\title{
Oxiesteróis: 0 seu Papel na Saúde e na Doença
}

\author{
M. Manuel Cruz Silva, João F. S. Carvalho, M. Luisa Sá e Melo*
}

O s oxiesteróis são derivados oxidados do colesterol, amplamente distribuídos no organismo humano. A diversidade de funções fisiológicas dos oxiesteróis continua a ser largamente investigada. Os oxiesteróis exercem efeitos citotóxicos, pró-apoptóticos e pró-inflamatórios que os implicam em diversas situações patológicas, nomeadamente a aterogénese e a neurodegenerescência. Por outro lado, os oxiesteróis têm vindo a revelar um potencial terapêutico interessante como compostos antitumorais e como promotores da osteogénese.

\section{INTRODUÇÃO}

Os oxiesteróis representam um grupo de derivados oxigenados do colesterol, o qual é composto de 3 regiões sub-estruturais: uma cadeia hidrocarbonada, também designada cadeia lateral, uma estrutura policíclica constituída por quatro anéis ( $A, B, C$ e D) e um grupo hidroxilo na posição 3 (Figura 1). Os oxiesteróis contêm um ou mais oxigénios adicionais na estrutura anelar ou na cadeia lateral e encontram-se distribuídos em fluidos e tecidos animais e em alimentos de origem animal.

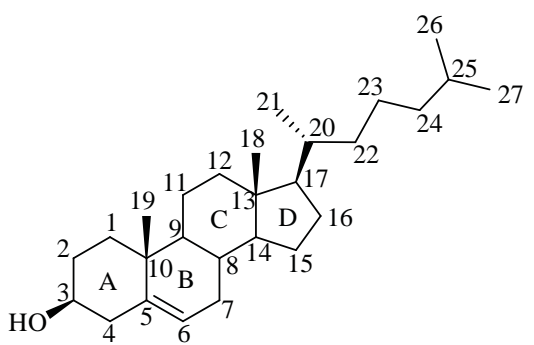

Figura 1 - Estrutura química do colesterol

In vivo, os oxiesteróis são formados por oxidação do colesterol, quer enzimática quer não-enzimática (Figura 2). Adicionalmente, os oxiesteróis podem entrar no organismo pela via alimentar.

A oxidação enzimática do colesterol envolve um conjunto de enzimas oxidativas. Os oxiesteróis mais representativos deste grupo

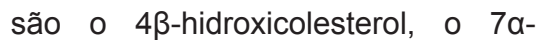

Faculdade de Farmácia, Universidade de Coimbra, Pólo das Ciências da Saúde, 3000-548, Coimbra, Portugal Centre for Neuroscience and Cell Biology, University of
Coimbra, Portugal

E-mail address: samelo@ff.uc.pt hidroxicolesterol e os oxiesteróis da cadeia lateral (Figura 2).

A oxidação não enzimática ocorre sobretudo ao nível da dupla ligação, a qual é susceptível ao ataque de radicais livres, embora possa ocorrer oxidação também na cadeia lateral.

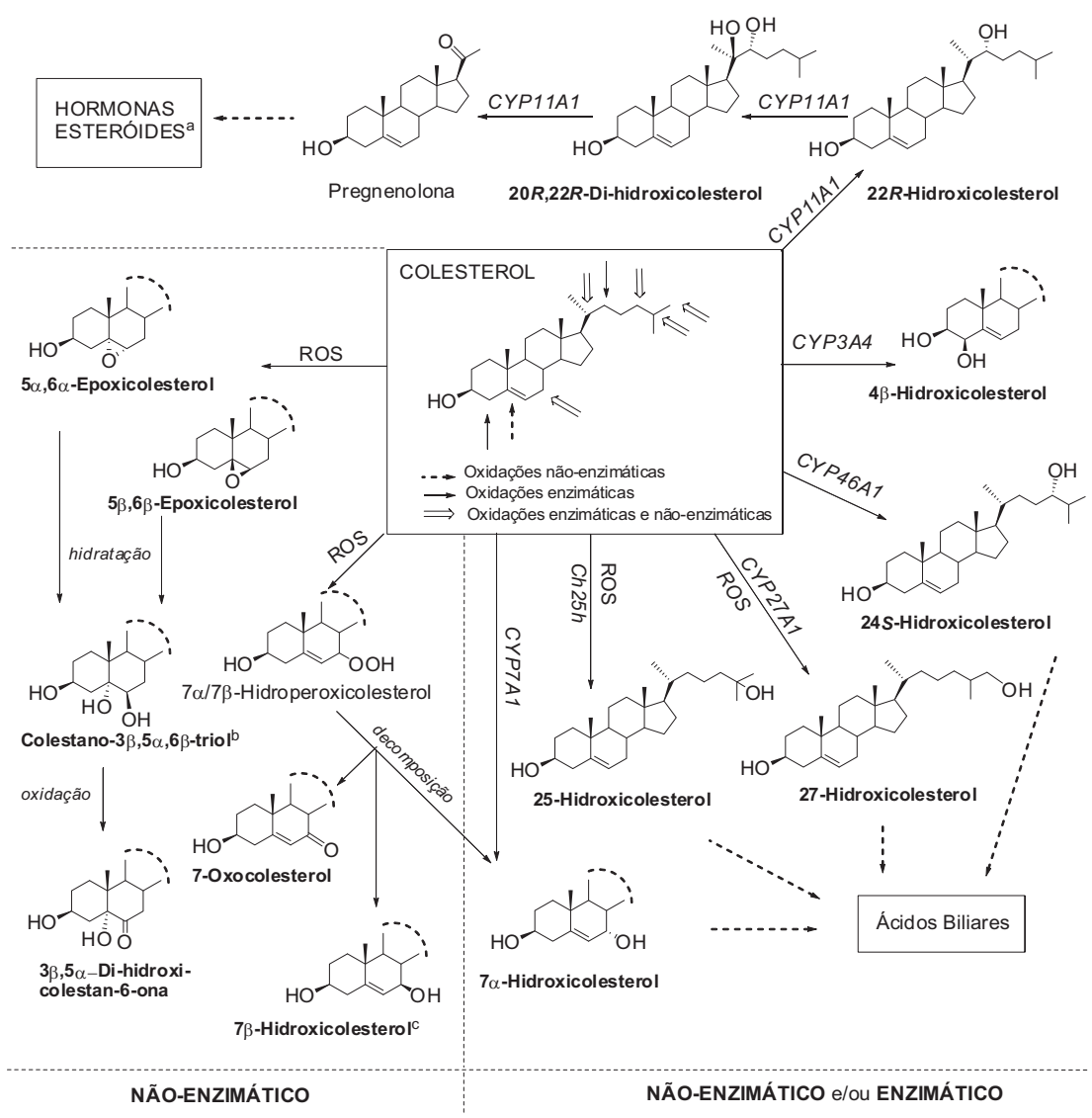

Figura 2 - Biossíntese dos oxiesteróis por auto-oxidação e por oxidação enzimática do colesterol [1-6]. ROS: espécies reactivas de oxigénio. Enzimas: CYP3A4: citocromo P 3 3A4; CYP7A1: colesterol $7 \alpha$-hidroxilase; CYP11A1: colesterol desmolase (P450scc); Ch25h: colesterol 25-hidroxilase; CYP27A1: esterol 27-hidroxilase; CYP46A1: colesterol 24-hidroxilase. a) Formação de hormonas esteróides de acordo com a via mais aceite de clivagem da cadeia lateral, embora outras vias tenham sido sugeridas [7]; b) a formação de colestano-3 $3,5 \alpha, 6 \beta$-triol pode também ser mediada in vivo através da biotransformação do 5,6-epoxicolesterol pela respectiva hidrolase [8]; c) a redução enzimática do 7-oxocolesterol pela 11ß-hidroxiesteróide desidrogenase tipo 1 (CYP11B1) foi descrita como uma via de síntese do $7 \beta$-hidroxicolesterol $[9,10]$, a qual pode contribuir para o rápido metabolismo do 7-oxocolesterol no fígado [11] 
A introdução de um oxigénio aumenta a velocidade de degradação do colesterol em produtos mais polares e facilita a saída da célula e posterior eliminação.

Os oxiesteróis são transportados no plasma pelas lipoproteínas, sobretudo pelas lipoproteínas de baixa densidade.

Os oxiesteróis são conhecidos desde há várias décadas. $\mathrm{O}$ isolamento do primeiro oxiesterol, o $7 \beta$ hidroxicolesterol, data de 1939 [12]. Todavia, apenas nos anos 70 os efeitos biológicos dos oxiesteróis começaram a ser conhecidos, nomeadamente, efeitos antiproliferativos [13] e de regulação da homeostase do colesterol [14]. Uma intensa investigação foi desencadeada desde então, procurando esclarecer o papel fisiológico dos oxiesteróis, bem como o seu envolvimento em algumas situações patológicas.

Devido à sua natureza anfifílica, os oxiesteróis atravessam as membranas celulares com maior facilidade que o colesterol. Assim, a formação de oxiesteróis in vivo, foi apontada como uma estratégia para eliminar o colesterol em excesso no organismo [15]. Por outro lado, alguns autores apontaram a formação não enzimática de oxiesteróis como sendo uma forma resultante do colesterol exercer uma actividade antioxidante in vivo $[16,17]$.

A formação enzimática de oxiesteróis está relacionada com a homeostase do colesterol [18]. Por um lado, constitui uma via de remoção do colesterol do organismo, transformando-o em derivados oxidados que posteriormente são convertidos em diversos compostos, nomeadamente ácidos biliares. Por outro lado, os oxiesteróis formados por via enzimática inibem a enzima hidroximetil glutaril coenzima A(HMG-CoA) redutase, enzima-chave da biossíntese do colesterol [19-21].

Porém, os oxiesteróis são mais do que produtos do metabolismo do colesterol. De facto, sendo uma parte deles formados por via enzimática e tendo alguns a capacidade de se ligarem a determinadas proteínas com elevada afinidade [22], é de esperar que a $\mathrm{Na}$ tureza Ihes tenha atribuído funções fisiológicas específicas.

Os oxiesteróis têm atraído atenção devido ao seu envolvimento num grande número de fenómenos biológicos, sendo cada vez mais considerados importantes mediadores fisiológicos dos efeitos do colesterol in vivo [2325]. Tem sido feito um grande esforço no sentido de identificar receptores celulares dos oxiesteróis e de os correlacionar com as funções dos oxiesteróis [22]

Os oxiesteróis formados enzimaticamente participam na biossíntese dos ácidos biliares [26] e das hormonas esteróides [27-29], actuando também como lípidos de sinalização que regulam a biossíntese do colesterol, o efluxo do colesterol celular, a recaptação de lipoproteínas e o tráfico intracelular do colesterol [24].

\section{OXiesteróis e Doença}

Os oxiesteróis têm sido implicados em diversas situações patológicas, em consequência das suas propriedades citotóxicas, oxidativas e pro-inflamatórias [30]. Para além do envolvimento dos oxiesteróis na aterosclerose e nas doenças neurodegenerativas, como se discute em seguida, outras patologias, como a doença macular degenerativa [31], cataratas e osteoporose [30], parecem também estar associadas à presença de oxiesteróis nos tecidos ou órgãos alvo.

\section{Aterosclerose}

Actualmente, é amplamente aceite o envolvimento da hipercolesterolémia na aterosclerose.

O $7 \beta$-hidroxicolesterol, o $5 \beta, 6 \beta$ epoxicolesterol e o 7-oxocolesterol encontram-se presentes na placa aterosclerótica em elevadas concentrações. Assim, o envolvimento dos oxiesteróis formados por reacções oxidativas não enzimáticas na aterosclerose tem sido muito estudado [32, 33].

Os oxiesteróis induzem apoptose em diversos tipos de células da vasculatura, existindo um elevado número de estudos acerca dos mecanismos sub- jacentes à morte celular [24, 34]. Sendo a morte celular um dos principais eventos no desenvolvimento do ateroma [35], a indução de apoptose pelos oxiesteróis pode ser um dos factores que contribuem para a aterosclerose. De igual forma, o envolvimento da peroxidação lipídica na aterogénese encontra-se amplamente documentado [36]. Os oxiesteróis, sendo uma consequência da acção dos radicais livres de oxigénio, são também prooxidativos. Estudos em ratos indicam que a administração de oxiesteróis conduz a um aumento da expressão e da actividade de enzimas antioxidantes e à redução das concentrações de glutatião total e reduzido, sugerindo uma interferência com os mecanismos de defesa antioxidante [37]. Noutro modelo, foi demonstrado que os oxiesteróis induzem a activação da NADPH-oxidase, a libertação de ácido araquidónico e a produção de anião superóxido, contribuindo para a oxidação mediada pelas lipoproteínas de baixa densidade [38].

As áreas da vasculatura sujeitas a uma lesão aterosclerótica encontramse num persistente estado de inflamação. Estudos in vitro revelaram que diversos oxiesteróis estimulam a produção de interleucina-8, uma quimiocina associada à inflamação [39] e aumentam a expressão e a actividade de uma fosfolipase [40], apontando, assim, para uma actividade pro-inflamatória dos oxiesteróis que contribui para a aterogénese.

Por fim, o envolvimento dos oxiesteróis na aterosclerose está ainda relacionado com a sua contribuição para a formação de células esponjosas. O 7-oxocolesterol favorece in vitro a diferenciação dos monócitos e a formação daquelas células [41], bem como a acumulação intracelular de lípidos polares [42].

Assim, o envolvimento dos oxiesteróis na aterogénese, embora controverso, não pode ser excluído e continuará a ser um importante tema de investigação [43].

\section{Doenças neurodegenerativas}

Os oxiesteróis têm sido implicados em doenças neurodegenerativas, como a 
doença de Parkinson, de Alzheimer e a esclerose múltipla [44] e diversas linhas de investigação têm procurado esclarecer os efeitos in vitro e in vivo dos oxiesteróis no cérebro, identificar possíveis alvos terapêuticos e identificar oxiesteróis que possam funcionar como biomarcadores de doenças neurodegenerativas.

O cérebro é o órgão do corpo humano mais rico em colesterol [45]. A síntese de colesterol no cérebro é muito elevada nas primeiras etapas da vida, passando na idade adulta a ser pouco significativa [46]. Dada a sua importância fisiológica neste órgão, os elevados níveis cerebrais de colesterol são mantidos por mecanismos homeostáticos altamente eficientes. Diversos estudos epidemiológicos in vitro apontam para uma relação entre o turnover do colesterol e algumas doenças neurodegenerativas. De facto, a hipercolesterolémia é apontada como um factor de risco para as doenças de Alzheimer e de Parkinson. Embora a síntese de novo do colesterol no cérebro adulto seja muito lenta, este tem de possuir um mecanismo de excreção do colesterol, prevenindo a sua acumulação, uma vez que a barreira hematoencefálica não tem capacidade de estabelecer trocas de colesterol com outros tecidos e fluidos. Assim, a oxidação enzimática do colesterol a 24S-hidroxicolesterol, o qual tem a capacidade de atravessar a barreira hematoencefálica, tem sido apontada como o principal mecanismo de eliminação do colesterol do cérebro [47-49].

Este oxiesterol parece desempenhar um papel neuroprotector. Estudos in vitro mostram que inibe a formação de peptídeos $A \beta$ [50] e estimula a actividade da $\alpha$-secretase, diminuindo a deposição do peptídeo $\beta$-amilóide [51]. Além disso o 24S-hidroxicolesterol é um potente ligando endógeno do receptor LXR (liver X receptor) e a activação deste receptor está relacionada com efeitos anti-amiloidogénicos e anti-inflamatórios [52, 53].

Todavia, efeitos pró-inflamatórios, através da indução da expressão das enzimas ciclo-oxigenase e fosfolipase A2 em células neuronais foram reportados para o 24S-hidroxicolesterol
[54]. Mais recentemente, foram descritos efeitos neurotóxicos do mesmo oxiesterol em modelos animais [55].

Por outro lado, o 27-hidroxicolesterol, não sendo produzido no cérebro, atravessa a barreira hematoencefálica e actua na regulação de diversas enzimas cerebrais. Estudos in vitro revelam que o 27-hidroxicolesterol está implicado na redução da actividade da proteína Arc, importante para a consolidação da memória [56] e diminui a actividade da $\alpha$-secretase [51]. Estudos ex vivo em doentes de Alzheimer revelaram um rácio aumentado de 27-hidroxicolesterol versus 24S-hidroxicolesterol [57]. Diversos autores apontam o 27-hidroxicolesterol como a chave para a relação epidemiológica entre hipercolesterolémia e doença de Alzheimer [59].

Outro derivado oxigenado do colesterol na cadeia lateral, o 25-hidroxicolesterol, exerce efeitos tóxicos no sistema nervoso central, nomeadamente, alteração da morfologia e apoptose de oligodendrócitos [58].

Adicionalmente, alguns oxiesteróis são directamente neurotóxicos e contribuem para o agravamento das doenças neurodegenerativas. $07 \beta$ hidroxicolesterol estimula a morte celular neuronal em concentrações nanomolares e diminui a actividade da a-secretase [60].

Diversos estudos apontam para níveis elevados de oxiesteróis em áreas do cérebro em estado de neuroinflamação após um dano excitotóxico. Por exemplo, o 7-oxocolesterol tem a capacidade de induzir morte neuronal por apoptose [61] e de despoletar a exocitose e a libertação de neurotransmissores nas áreas afectadas, agravando a neuroinflamação [62].

\section{Oxiesteróis com Potencial Activl- DAde Terapêutica}

\section{Cancro}

A potencial actividade antitumoral dos oxiesteróis é conhecida de há muito. $A$ citotoxicidade do $7 \beta$-hidroxicolesterol foi referida na década de $70[13,14$, 63] e tem vinda a ser estudada para diferentes oxiesteróis em diversas li- nhas celulares tumorais e não-tumorais. Além disso, os oxiesteróis têm sido usados na medicina popular [6466], o que corrobora a sua potencial utilidade como agentes antitumorais.

Diversos estudos descrevem o efeito citotóxico dos oxiesteróis em linhas celulares da vasculatura, procurando relacionar esse efeito com a aterosclerose [24, 32-34, 42]. Por outro lado, muitos estudos reportam os efeitos citotóxicos destes compostos em linhas celulares de cancro, sendo estes dependentes da estrutura do oxiesterol, da sua concentração, da linha celular em estudo e do tempo de incubação [34, 67].

A capacidade de induzir morte celular, sobretudo por apoptose, tem sido demonstrada em diversas linhas celulares tumorais e não-tumorais [44, 67-69], embora a morte celular por necrose tenha também sido demonstrada em alguns casos [70-72].

Em geral, os oxiesteróis oxigenados no núcleo esteróide são mais citotóxicos e mais potentes na indução de apoptose do que os oxiesteróis oxigenados na cadeia lateral [73-76], os quais exercem o seu efeito sobretudo através da inibição da síntese do colesterol.

Adicionalmente, diversos análogos oxigenados do colesterol têm sido isolados de organismos marinhos, alguns dos quais foram sintetizados e os seus efeitos citotóxicos estudados [77-84] (Figura 3).

O nosso grupo sintetizou e avaliou 8 oxiesteróis endógenos em células HT29 (de adenocarcinoma do cólon) e ARPE-19 (do epitélio pigmentado da retina). Pela primeira vez, a actividade citotóxica destes derivados oxigenados nos anéis $A$ e $B$ foi estudada sob as mesmas condições experimentais, permitindo uma comparação da sua potência e selectividade [85]. Adicionalmente, a influência da posição e da estereoquímica do grupo epóxido no anel A ou B, bem como da presença de outros grupos oxigenados, foram estudadas após síntese químio-enzimática de uma biblioteca de epóxidos epimericamente puros [86]. A síntese de um conjunto mais vasto de esterói- 
des oxigenados permitiu tirar conclusões acerca do tipo de cadeia lateral e tipo de substituintes no anel B capazes de fornecer oxiesteróis sintéticos com maior potência e selectividade para células tumorais [85]. As relações estrutura-actividade encontram-se sumariadas na Figura 4.

Adicionalmente, os oxiesteróis aumentam a sensibilidade das células tumorais à radioterapia [87, 88] e à quimioterapia [88]. No nosso grupo, foi estudado o efeito do colestano$3 \beta, 5 \alpha, 6 \beta$-triol na citotoxicidade da doxorubicina numa linha celular tumoral e noutra não-tumoral, tendo-se verificado que uma concentração não tóxica do oxiesterol consegue diminuir para metade $0 \mathrm{IC}_{50}$ da doxorubicina nas células tumorais sem afectar as células não-tumorais [85].

É conhecido que os oxiesteróis estão envolvidos quer na proliferação quer na morte celular. Todavia, os mecanismos responsáveis pela citotoxicidade dos oxiesteróis estão longe de estar esclarecidos.

Os oxiesteróis mais comuns interferem com a estrutura da membrana celular e com receptores celulares [69, 89], inibem a biossíntese do colesterol [14, 24, 90] e do DNA [91, 92], podendo estes efeitos contribuir para a sua citotoxicidade.

Por outro lado, uma vez que o colesterol é essencial para a progressão da mitose [93], a modulação da síntese do colesterol e a competição com o colesterol para a membrana celular, podem justificar a citotoxicidade dos oxiesteróis [93, 94].

Os efeitos dos oxiesteróis na biofísica membranar, alterando a fluidez e a permeabilidade, interferindo com as proteínas membranares e, deste
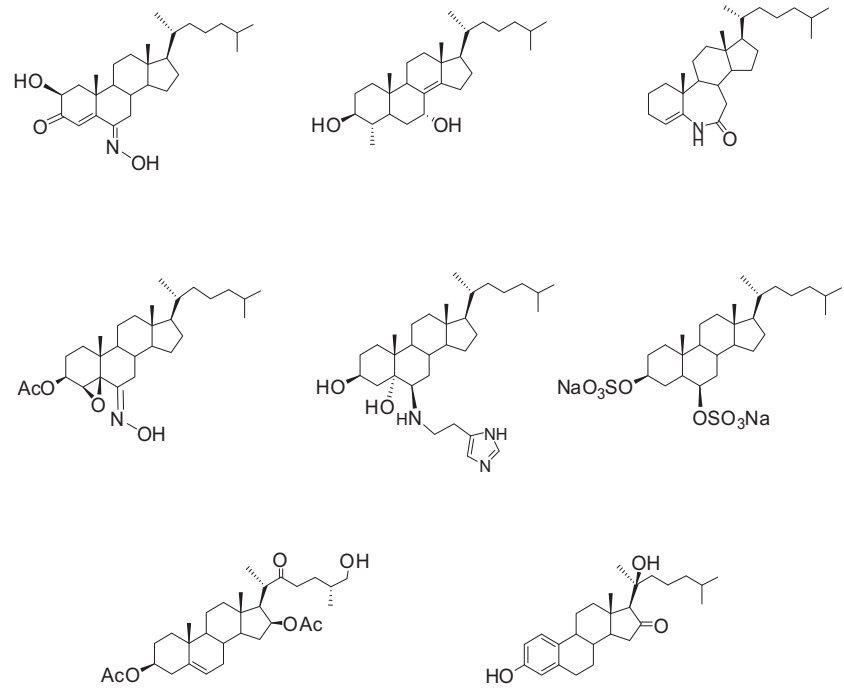

Figura 3 - Estruturas de novos oxiesteróis recentemente sintetizados com citotoxicidade relevante

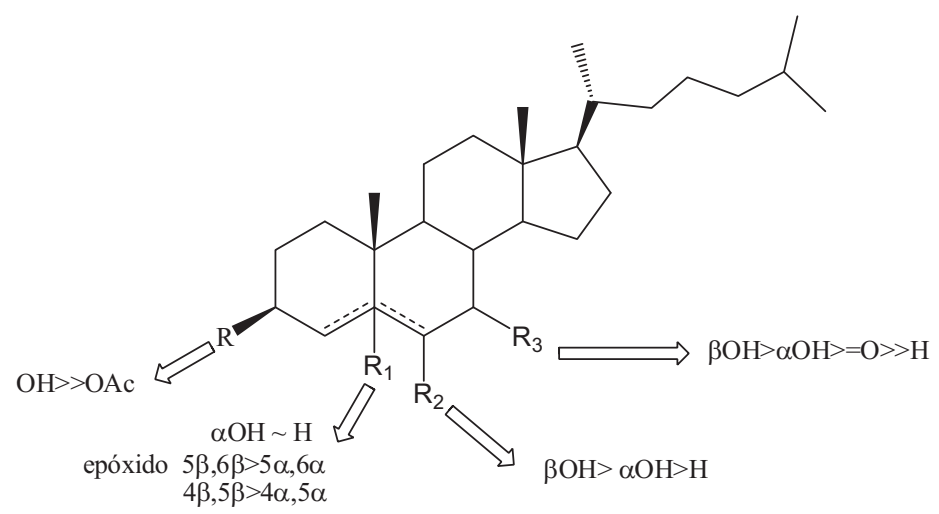

Figura 4 - Representação gráfica das relações estrutura - actividade citotóxica dos oxiesteróis, com base nos resultados de $\mathrm{IC}_{50}$ em células tumorais modo, com o funcionamento da célula, são também factores a ter em conta no esclarecimento dos mecanismos de citotoxicidade dos oxiesteróis e na explicação da diferente susceptibilidade das linhas celulares aos efeitos tóxicos dos oxiesteróis [69, 95-97].

\section{Medicina regenerativa}

Os oxiesteróis participam na morfogénese [98] através da via de sinalização de Hedgehog [99, 100], a qual tem um papel chave no desenvolvimento pósembrionário, na homeostase dos tecidos adultos e na fisiologia das células estaminais [101].

A activação da via de sinalização de Hedgehog em células estaminais pelo 20S-hidroxicolesterol resulta na inibição da adipogénese e no favorecimento da osteogénese [102]. Estudos in vitro e em modelos animais demonstraram que o 22R- e o 22S-hidroxicolesterol induzem a diferenciação osteoblástica de células estaminais, bem como a formação de tecido ósseo in vivo [103].

Estes efeitos fisiológicos dos oxiesteróis têm grande importância na formação, homeostase e reparação dos tecidos e revelam um potencial terapêutico que começa a ser explorado. Recentemente, uma biblioteca de compostos estruturalmente relacionados com os oxiesteróis foi sintetizada e novas moléculas promotoras da osteogénese foram identificadas [104].

\section{Perspectivas Futuras}

Os oxiesteróis são importantes reguladores dos rafts lipídicos [105, 106], estão envolvidos na inflamação [39, 40], possuem propriedades imunossupressoras [107-109], foram referidos como antifúngicos [110] e antivirais [111], continuando assim a ser muito estudados [3, 112].

A correlação das estruturas moleculares dos oxiesteróis com as suas actividades biológicas permite estabelecer uma relação entre a química e a química biológica destes compostos e permitirá no futuro desenvolver estratégias terapêuticas baseadas nos oxiesteróis e seus alvos celulares. 


\section{REFERÊNCIAS}

[1] L. L. Smith, Chem. Phys. Lipids 44 (1987) 87-125.

[2] M. Yamaguchi, Y. Endo, M. Shimizu, K. Yamamoto, S. Yamada, K. Shudo, Biol. Pharm. Bull. 20 (1997) 10441046.

[3] I. Björkhem, U. Diczfalusy, Arterioscler. Thromb. Vasc. Biol. 22 (2002), 734-742.

[4] A. V. Antonchick, V. N. Zhabinskii, V. A. Khripach, Russ. J. Bioorg. Chem. 33 (2007) 275-287.

[5] R. C. Murphy, K. M. Johnson, J. Biol. Chem. 283 (2008) 15521-15525.

[6] A. J. Brown, W. Jessup, Mol. Aspects Med. 30 (2009) 111-122.

[7] S. Lieberman, Y. Y. Lin, J. Steroid Biochem. Mol. Biol. 78 (2001) 1-14.

[8] J. W. Newman, C. Morisseau, B. D. Hammock, Prog. Lipid Res. 44 (2005) 1-51.

[9] R. A. S. Schweizer, M. Zürcher, Z. Balazs, B. Dick, A. Odermatt, J. Biol. Chem. 279 (2004) 18415-18424.

[10] M. Hult, B. Elleby, N. Shafqat, S. Svensson, A. Rane, H. Jörnvall, L. Abrahmsen, U. Oppermann, Cell. Mol. Life Sci. 61 (2004) 992-999.

[11] M. A. Lyons, S. Samman, L. Gatto, A. J. Brown, J. Lipid Res. 40 (1999) 1846-1857.

[12] G. A. Haslewwod, Biochem. J. 33 (1939) 709-712.

[13] H. W. Chen, A. A. Kandutsch, C. Waymouth, Nature 251 (1974) 419-421.

[14] A. A. Kandutsch, H. W. Chen, H.-J. Heiniger, Science 201 (1978) 498501.

[15] E. Lund, O. Andersson, J. Zhang, A. Babiker, G. Ahlborg, U. Diczfalusy, K. Einarsson, J. Sjövall, I. Björkhem, Arterioscler. Thromb. Vasc. Biol. 16 (1996) 208-212.

[16] L. L. Smith, Free Radical Biol. Med. 11 (1991) 47-61.

[17] H. Girao, C. Mota, P. Pereira, Curr. Eye Res. 18 (1999) 448-454.

[18] S. Gill, R. Chow, A. J. Brown, Prog. Lipid Res. 47 (2008) 391-404.

[19] A. Radhakrishnan, Y. Ikeda, H. J. Kwon, M. S. Brown, J. L. Goldstein, Proc. Natl. Acad. Sci. USA 104 (2007) 6511-6518.

[20] L.-P. Sun, J. Seemann, J. L. Goldstein, M. S. Brown, Proc. Natl. Acad. Sci. USA 104 (2007) 6519-6526.

[21] Y. Wang, P. M. Rogers, C. Su, G. Varga, K. R. Stayrook, T. P. Burris, J. Biol. Chem. 283 (2008) 26332-26339.
[22] V. M. Olkkonen, Lipids Insights 2 (2008) 1-9.

[23] R. C. Murphy, K. M. Johnson, J. Biol. Chem. 283 (2008) 15521-15525.

[24] G. J. J. Schroepfer, Physiol. Rev. 80 (2000) 361-554.

[25] I. Björkhem, J. Clin. Invest. 110 (2002) 725-730.

[26] M. Fuchs, Am. J. Physiol. Gastrointest. Liver Physiol. 284 (2003) G551-G557.

[27] S. Lieberman, Y. Y. Lin, J. Steroid Biochem. Mol. Biol. 78 (2001) 1-14.

[28] L. K. Christenson, J. M. McAllister, K. O. Martin, N. B. Javitt, T. F. Osborne, J. F. Strauss, J. Biol. Chem. 273 (1998) 30729-30735.

[29] S. R. King, A. A. Matassa, E. K. White, L. P. Walsh, Y. Jo, R. M. Rao, D. M. Stocco, M. E. Reyland, J. Mol. Endocrinol. 32 (2004) 507-517.

[30] G. Schwartsmann, M. J. Ratain, G. M. Cragg, J. E. Wong, N. Saijo, D. R. Parkinson, Y. Fujiwara, R. Pazdur, D. J. Newman, R. Dagher, L. Di Leone, J. Clin. Oncol. 20 (2002) 47s-59s.

[31] I. R. Rodriguez, I. M. Larrayoz, J. Lipid Res. 51 (2010) 2847-2862.

[32] A. J. Brown, W. Jessup, Atherosclerosis 142 (1999) 1-28.

[33] G. Poli, B. Sottero, S. Gargiulo, G. Leonarduzzi, Mol. Aspects Med. 30 (2009) 180-189.

[34] S. R. Panini, M. S. Sinensky, Curr. Op. Lipidol. 12 (2001) 529-533.

[35] E. Falk, J. Am. Coll. Cardiol. 47 (2006) 7-12.

[36] R. Stocker, J. F. Keaney, Physiological Rev. 84 (2004) 1381-1478.

[37] R. Ringseis, K. Eder, Int. J. Vitam. Nutr. Res. 74 (2004) 86-92.

[38] M. Rosenblat, M. Aviram, Atherosclerosis 160 (2002) 69-80.

[39] S. Lemaire-Ewing, C. Prunet, T. Montange, A. Vejux, A. Berthier, G. Bessede, L. Corcos, P. Gambert, D. Neel, G. Lizard, Cell Biol. Toxicol. 21 (2005) 97-114.

[40] V. Antonio, B. Janvier, A. Brouillet, M. Andreani, M. Raymondjean, Biochem. J. 376 (2003) 351-360.

[41] J. M. Hayden, L. Brachova, K. Higgins, L. Obermiller, A. Sevanian, S. Khandrika, P. D. Reaven, J. Lipid Res. 43 (2002) 26-35.

[42] A. Vejux, E. Kahn, F. Ménétrier, T. Montange, J. Lherminier, J.-M. Riedinger, G. Lizard, Histochem. Cell. Biol. 127 (2007) 609-624.

[43] G. Poli, B. Sottero, S. Gargiulo, G. Leonarduzzi, Molec. Asp. Med. 30 (2009) 180-189.
[44] A.Vejux,; G.Lizard, Mol. Aspects Med. 30 (2009) 153-170

[45] I. Bjorkhem, S. Meaney, Arterioscl. Thromb. Vasc. Biol.24(2004)806-815.

[46] J. M. Dietschy, S. D. Turley, J. Lipid Res. 45 (2004) 1375-1397

[47] D. Lutjohann, O. Breuer, G. Ahlborg, I. Nennesmo, A. Siden, U. Diczfalusy, I. Bjorkhem, Proc. Natl. Acad. Sci. USA 93 (1996) 9799-9804.

[48] I. Bjorkhem, D. Lutjohann, O. Breuer, A. Sakinis, A. Wennmalm, J. Biol. Chem. 272 (1997) 30178-30184.

[49] D. W. Russell, R. W. Halford, D. M. O. Ramirez, R. Shah, T. Kotti, Ann. Rev. Biochem. 78 (2009) 1017-1040.

[50] J. Brown, C. Theisler, S. Silberman, D. Magnuson, N. Gottardi-Littell, J. M. Lee, D. Yager, J. Crowley, K. Sambamurti, M. M. Rahman, A. B. Reiss, C. B. Eckman, B. Wolozin, J. Biol. Chem. 279 (2004) 34674-34681.

[51] D. Famer, S. Meaney, M. Mousavi, A. Nordberg, I. Björkhem, M. Crisby, Biochem. Biophys. Res. Commun. 359 (2007) 46-50.

[52] B. A. Janowski, M. J. Grogan, S. A. Jones, G. B. Wisely, S.A. Kliewer, E. J. Corey, D. J. Mangelsdorf, Proc. Natl. Acad. Sci. USA 96 (1999) 266-271.

[53] G. Cao, K. R. Bales, R. B. Demattos, S. M. Paul, Curr. Alzheimer Res. 4 (2007) 179-184.

[54] P. Alexandrov, J. G. Cui, Y. H. Zhao, W. J. Lukiw, Neuroreport 16 (2005) 909913.

[55] S. Zhao, W. Liao, N. Xu, H. Xu, C. Yu, X. Liu C. Li, Neuroscience 164 (2009) 398-403.

[56] L. Mateos, S. Akterin, F. J. Gil-Bea, S. Spulber, A. Rahman, I. Bjorkhem, M. Schultzberg, A. Flores-Morales, A. Cedazo-Minguez, Brain Pathology 19 (2009) 69-80.

[57] M. Heverin, N. Bogdanovic, D. Lutjohann, T. Bayer, I. Pikuleva, L. Bretillon, U. Diczfalusy, B. Winblad, I. Bjorkhem, J. Lipid Res. 45 (2004) 186-193.

[58] A. Trousson, S. Bernard, P. X. Petit, P. Liere, A. Pianos, K. El Hadri, J. M. Lobaccaro, M. Said Ghandour, M. Raymondjean, M. Schumacher, C. Massaad., J. Neurochem. 109 (2009) 945-958.

[59] M. Stefani, G. Liguri, Curr. Alzheimer Res. 6 (2009) 15-29.

[60] T. J. Nelson, D. L. Alkon, J. Biol. Chem. 280 (2005) 7377-7387.

[61] E. R. Jang, C. S. Lee, Neurochem. Int. 58 (2011) 52-59. 
[62] M. T. Ma, J. Zhang, A. A. Farooqui, P. Chen, W. Y. Ong, Neurosc. Lett. 476 (2010) 36-41.

[63] S. K. Peng, C. B. Taylor, P. Tham, N. T. Werthessen, B. Mikkelson, Arch. Pathol. Lab. Med. 102 (1978) 57-61.

[64] K. P Cheng, H. Nagano, B. Luu, G. Ourisson, J. P. Beck, J. Chem. Res. 217 (1977) 2501-2521.

[65] J. Nagano, J. P. Poyser, K. P Cheng, B. Luu, G. Ourisson, J. P Beck, J. Chem. Res. 218 (1977) 2522-2571.

[66] M. Zander, K. Patrick, L. Bang, G. Ourisson, J. Chem. Res. 219 (1977) 2572-2584.

[67] L. Ryan, Y. C. O'Callaghan, N. M. O'Brien, Curr. Nutr. Food Sci. 1 (2005) 41-51.

[68] T. Wielkoszynski, K. Gawron, J. Strzelczyk, P. Bodzek, M. ZalewskaZiob, G. Trapp, M. Srebniak, A. Wiczkowski, BioEssays 28 (2006) 387398.

[69] S. Lordan, J. J. Mackrill, N. M. O'Brien, J. Nutr. Biochem. 20 (2009) 321-336.

[70] A. Rimner, S. Al Makdessi, H. Sweidan, J. Wischhusen, B. Rabenstein, K. Shatat, P. Mayer, L. Spyridopoulos, Free Radic. Biol. Med. 38 (2005) 535544.

[71] G. Lizard, S. Monier, C. Cordelet, L. Gesquiere, V. Deckert, S. Gueldry, L. Lagrost, P. Gambert, Arterioscler. Thromb. Vasc. Biol. 19 (1999) 11901200.

[72] Y. C. O'Callaghan, J. A. Woods, N. M. O'Brien, Toxicol. in Vitro 16 (2002) 245-251.

[73] S. Lemaire-Ewing, C. Prunet, T. Montange, A. Vejux, A. Berthier, G. Bessède, L. Corcos, P. Gambert, D. Néel, G. Lizard, Cell Biol. Toxicol. 21 (2005) 97-114.

[74] G. Lizard, V. Deckert, L. Dubrez, M. Moisant, P. Gambert, L. Lagrost, Am. J. Pathol. 148 (1996) 1625-1638.

[75] J. Y. Chang, L.-Z. Liu, Curr. Eye Res. 17 (1998) 95-103.

[76] A. J. O'Sullivan, Y. C. O'Callaghan, J. A. Woods, N. M. O'Brien, J. Appl. Toxicol. 23 (2003) 191-197.

[77] N. Deive, J. Rodríguez, C. Jiménez,
J. Med. Chem. 44 (2001) 2612-2618.

[78] L. He, Y. Liu, J. Shi, Q. Pei, Steroids 71 (2006) 476-483.

[79] N. M. Krstic, M. S. Bjelakovic, Z. Zizak, M. D. Pavlovic, Z. D. Juranic, V. D. Pavlovic, Steroids 72 (2007) 406-414.

[80] J. Poza, M. Rega, V. Paz, B. Alonso, J. Rodríguez, N. Salvador, A. Fernández, C. Jiménez, Bioorg. Med. Chem. 15 (2007) 4722-4740.

[81] P. de Medina, M. R. Paillasse, B. Payré, S. Silvente-Poirot, M. Poirot, J. Med. Chem. 52 (2009) 7765-7777.

[82] J. Cui, H. Wang, Y. Huang, Y. Xin, A. Zhou, Steroids 74 (2009) 1057-1060.

[83] M. A. Fernández-Herrera, H. LópezMuñoz, J. M. V. Hernández-Vázquez, M. López-Dávila, M. L. EscobarSánchez, L. Sánchez-Sánchez, B. M. Pinto, J. Sandoval-Ramírez, Bioorg. Med. Chem. 18 (2010) 2474-2484.

[84] P. Bunyathaworn, S. Boonananwong, B. Kongkathip, N. Kongkathip, Steroids 75 (2010) 432-444.

[85] J. F. S. Carvalho, M. M. Cruz Silva, J. N. Moreira, S. Simões, M. L. Sá e Melo, J. Med. Chem. 53 (2010) 76327638.

[86] J. F. S. Carvalho, M. M. Cruz Silva, J. N. Moreira, S. Simões, M. L. Sá e Melo, J. Med. Chem. 52 (2009) 40074019.

[87] K. G. David, B. F. Jim, P. S. Hans, F. S. Mark, S. Fritz, Photochem. Photobiol. 54 (1991) 717-723.

[88] J. W. Hyun, V. Holl, D. Weltin, P. Dufour, B. Luu, P. Bischoff, Anticancer Res. 22 (2002) 943-948.

[89] V. M. Olkkonen, R. Hynynen, Mol. Aspects Med. 30 (2009) 123-133.

[90] A. Radhakrishnan, Y. Ikeda, H. Kwon, M. Brown, J. Goldstein, Proc. Natl. Acad. Sci. USA 104 (2007) 65116518.

[91] M. Astruc, S. Roussillon, R. Defay, B. Descomps, A. C. de Paulet, Biochim. Biophys. Acta 763 (1983) 11-18.

[92] C. Ishimaru, Y. Yonezawa, I. Kuriyama, M. Nishida, H. Yoshida, Y. Mizushina, Lipids 43 (2008) 373-382.

[93] C. Fernández, M. V. T. Lobo, D. Gómez-Coronado, M. A. Lasunción, Exp. Cell Res. 300 (2004) 109-120.
[94] M. S. Brown, J. L. Goldstein, J. Biol. Chem. 249 (1974) 7306-7314.

[95] J. B. Massey, H. J. Pownall, Biochemistry 45 (2006) 10747-10758.

[96] D. Marsh, Biochim. Biophys. Acta 1778 (2008) 1545-1575.

[97] C. Yuan, R. J. O'Connell, R. F. Jacob, R. P. Mason, S. N. Treistman, J. Biol. Chem. 282 (2007) 7276-7286.

[98] N. B. Javit, Steroids 73 (2008) 149157.

[99] J. R. Dwyer, N. Sever, M. Carlson, S. F. Nelson, P. A. Beachy, F. Parhami, J. Biol. Chem. 282 (2007) 89598968.

[100] H. T. Kha, B. Basseri, D. Shouhed, J. Richardson, S. Tetradis, T. J. Hahn, F. Parhami, J. Bone Miner. Res. 19 (2004) 830-840.

[101] F. Simpson, M. C. Kerr, C. Wicking, Mechan. Develop. 126 (2009) 279288.

[102] J. R. Dwyer, N. Sever, M. Carlson, S. F. Nelson, P. A. Beachy, F. Parhami, J. Biol. Chem. 282 (2007) 8959-8968.

[103] T. L. Aghaloo, C. M. Amantea, C. M. Cowan, J. A. Richardson, B. M. Wu, F. Parhami, S. Tetradis, J. Orthop. Res. 25 (2007) 1488-1497.

[104] F. Parhami, M. E. Jung, K. Nguyen, D. Yoo, W.-K. Kim, Un. States Patent Applic. US 2011/0008297 A1

[105] J. B. Massey, H. J. Pownall, Biochemistry 44 (2005) 10423-10433.

[106] J. B. Massey, H. J. Pownall, Biochemistry 45 (2006) 10747-10758.

[107] C. Moog, B. Luu, J.-P. Beck, L. Italiano, P. Bischoff, Int. J. Immunopharmacol. 10 (1988) 511-518.

[108] C. Moog, Y. H. Ji, C. Waltzinger, B. Luu, P. Bischoff, Immunology $\mathbf{7 0}$ (1990) 344-350.

[109] P. L. Bischoff, V. Holl, D. Coelho, P. Dufour, B. Luu, D. Weltin, Curr. Med. Chem. 7 (2000) 693-713.

[110] J. M. Brunel, C. Loncle, N. Vidal, M. Dherbomez, Y. Letourneux, Steroids 70 (2005), 907-912.

[111] C. Moog, A. M. Aubertin, A. Kirn, B. Luu, Antivir. Chem. Chemother. 9 (1998) 491-496.

[112] C. Garenc, P. Julien, E. Levy, Free Rad. Res. 44, (2010) 47-73.

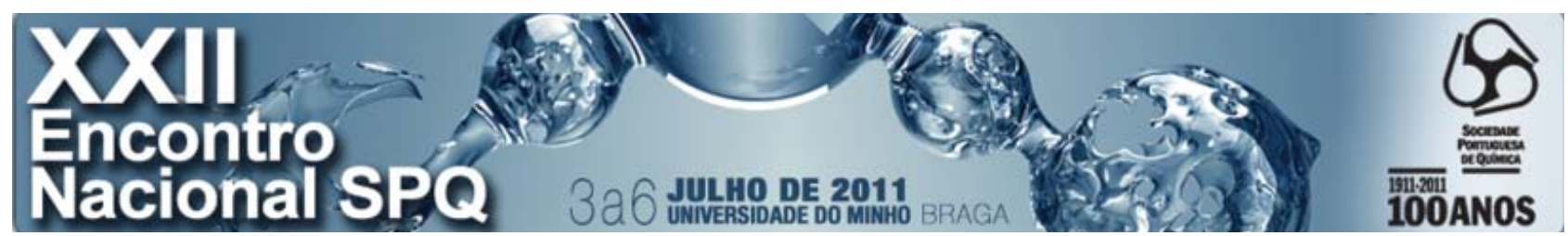

